\title{
Similarities and Differences in Cellular Processing of Biologically Relevant Nanoparticles
}

\author{
Burcu Kepsutlu ${ }^{1}$ and James McNally ${ }^{2}$ \\ ${ }^{1}$ Varex Imaging, Walluf, Hessen, Germany, ${ }^{2}$ Helmholtz Zentrum Berlin, Berlin, Berlin, Germany
}

\begin{abstract}
Nanoparticles are endocytosed and trafficked within cells by selecting one or more of the cell's intrinsic processing pathways. However, general rules which drive this selection have not yet been defined. Therefore, each nanoparticle has to be investigated separately to determine its endocytosis and intracellular trafficking pathways. By using cryo SXT to investigate the cellular interaction of two different nanoparticles, we have uncovered some general rules about nanoparticle interactions with cells. Our results highlight not only the importance of cryo-SXT for nanoparticle investigation in the medical field, but also demonstrate that a well-controlled analysis makes it possible to discern general rules about cell-nanoparticle interactions.
\end{abstract}

\section{Introduction}

We are constantly exposed to nanoparticles in our daily life. These nanoparticles either arise as byproducts of various industrial processes or they are specifically developed for our use. For example, in nanomedicine, nanoparticles are designed for drug delivery and/or diagnosis of disease. In order to synthesize nanoparticles specifically targeted to diseased cells or organelles, it is essential to understand the cellular interactions of the nanoparticle. Such an investigation is also vital to determine whether the nanoparticles reach their target, whether they localize to other unintended locations, whether they are eventually excreted, and whether they will induce unwanted side effects. For all of these reasons, the behavior and fate of nanoparticles in biological systems have been studied intensely. However, general rules which drive this selection have not yet been defined. Therefore, each nanoparticle has to be investigated separately to determine its endocytosis and intracellular trafficking pathways.

In this study, cellular interactions of two different biologically relevant nanoparticles, namely dendritic polyglycerol sulfate coated gold nanoparticles (dPGS-AuNP's) and polyethyleneimine coated gold nanoparticles (PEI-AuNP's), have been investigated using cryo soft X-ray tomography (cryo SXT) to determine if similar rules about their cellular interactions can be identified ${ }^{1}$. Each nanoparticle had the same $50 \mathrm{~nm}$ core size and each was used at the same concentration $(0.13 \mathrm{nM})$, for the same incubation period and in the same cell type (A549 lung epithelial cells). The only difference between the treatments was the nanoparticle coating. The dPGS coating is a highly sulfated, negatively charged polymer, while the PEI coating is a highly aminated, positively charged polymer. dPGS AuNP's are good candidates for the diagnosis and treatment of inflammation, and PEI AuNP's are promising agents for targeted gene delivery.

Cryo SXT is well suited to the analysis of gold nanoparticle uptake by cells because it provides the ability to image endocytic compartments of near-native state cells in 3D and to track nanoparticles relative to these compartments at $\sim 40 \mathrm{~nm}$ spatial resolution without a need for staining, chemical fixation or slicing.

\section{Results and Discussion}


$\underline{\text { Similarities in cellular interactions for the two nanoparticle types }}$

Using cryo SXT, we observed a number of similarities in the cellular uptake and processing of the dPGSAuNP's and the PEI-AuNP's (Fig. 1), even though their coatings were different. Specifically, we found that both types of nanoparticles were endocytosed by membrane ruffles $(\sim 0 \%)$ and secondarily by coated pits $(\sim 20 \%)$. Subsequently, both types of nanoparticles were found in endosomes, multivesicular bodies, lysosomes and free in the cytoplasm.

We also found that both types of nanoparticles induced similar changes in the organelle content of cells ${ }^{2}$. Specifically, we observed that nanoparticle uptake induced significant increases in the number of mitochondria and endosomes and significant decreases in the number of lipid droplets and multivesicular bodies. Some of these changes are similar to those observed in cells after nutrient deprivation or exercise ${ }^{3}$, and therefore may indicate that nanoparticle uptake requires large energy expenditures by the cell. Note that such a cytoplasmic remodeling in response to nanoparticles had not been detected before and was enabled here by the ability of cryo SXT to visualize all organelles in nearly an entire cell.

Differences in cellular interactions for the two nanoparticle types

We also observed some differences in the cellular responses between the two types of nanoparticles, and these likely reflect the chemical differences in the coating. For example, we found about 5x more PEIAuNP's in the cytoplasm compared to dPGS-AuNP's. This is consistent with PEI's property to promote cytoplasmic escape from membrane-bound organelles ${ }^{4}$. Perhaps as a consequence of this higher cytoplasmic concentration, we found some PEI-AuNP's in the nucleus, but no dPGS-AuNP's in the nucleus.

The dPGS-AuNP's were also found in some locations where there were no PEI-AuNP's, namely within autophagic vacuoles and lipid droplets. The lipid droplet location was especially interesting, as very few nanoparticles had ever been observed before in this compartment. This is also potentially a benefit of cryo SXT which enables better visualization of lipid droplet interiors compared to electron microscopy in which the lipid droplets become very densely stained.

\section{Conclusion}

In sum, a number of similar patterns were found in endocytosis, intracellular trafficking and morphological effects with the two types of nanoparticles analyzed. The differences, which were observed between the two nanoparticles, were consistent with their specific coating, and were therefore not entirely surprising. These observations suggest that the behavior of biologically relevant nanoparticles might become more predictable in the future by further application of systematic studies such as this in which the independent variable is restricted to only one, such as the surface coat or the concentration. 

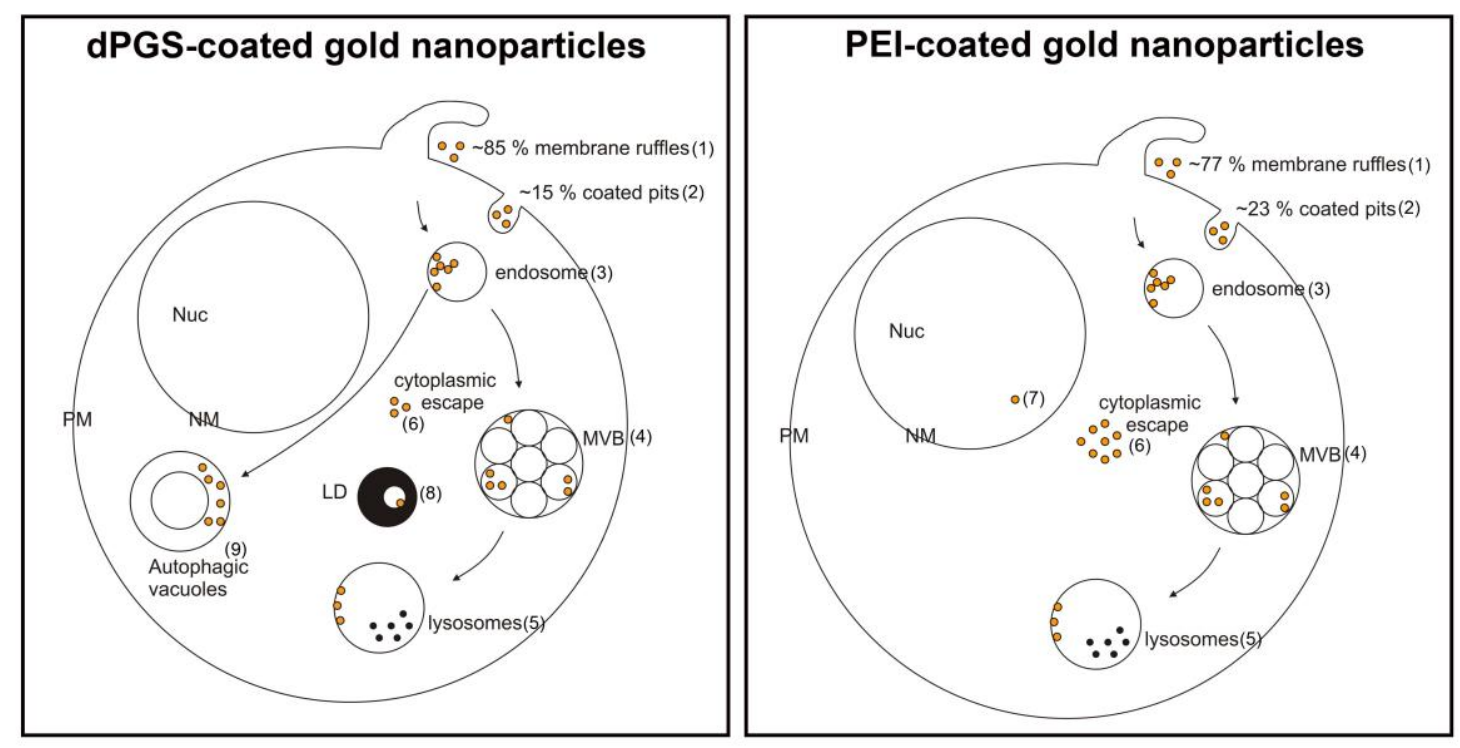

Figure 1. Despite different coatings, the two nanoparticles are endocytosed similarly $(1,2)$ and both are found in endosomes (3), multivesicular bodies (4), lysosomes (5) and free in the cytoplasm (6). Differences are in nuclear (7), lipid droplet (8) and autophagic vacuole (9) localization.

References

1. Kepsutlu, B. (2019) Interaction of biologically relevant nanoparticles with cells studied by cryo soft Xray tomography. PhD dissertation. Humboldt University Berlin.

2. Kepsutlu, B. et al. (2020) Cells undergo major changes in the quantity of cytoplasmic organelles after uptake of gold nanoparticles with biologically relevant surface coatings. ACS Nano 14:2248-2264.

3. Aon, M.A. et al. (2014) Mitochondrial and cellular mechanisms for managing lipid excess. Front. Physiol. 5: 282.

4. Bus, T. et al. (2018) The great escape: how cationic polyplexes overcome the endosomal barrier. $J$. Mater. Chem. B 6:6904-6918. 